\title{
Droplet impact on water layers: post-impact analysis and computations
}

\author{
By R. Purvis and F. T. Smith \\ Mathematics Department, University College, Gower Street, \\ London WC1E 6BT, UK \\ (frank@math.ucl.ac.uk)
}

An inviscid model of droplet impact into a water layer is examined analytically for small times just after impact and computationally for order-one distortion times. Various layer depths are considered, as are surface-tension effects. Good correspondence is found between analysis, computation and experiments.

Keywords: droplet; impact; splashing; small times

\section{Introduction}

The high-speed impact of a single water droplet onto a previously undisturbed layer of water has a range of applications; for example, in the chocolate manufacturing, spray-coating and aeronautics industries and particularly in regard to aircraft icing. When flying through clouds at or below freezing temperature, aircraft can accrete ice on forward-facing parts of the aircraft, most crucially on the leading edge of a wing, on the tail and around the engine intakes. Supercooled water droplets suspended in the cloud impact upon the aircraft. After impact, depending on temperature and speed, water can turn to ice immediately or it can spread further aft down the wing before freezing. These different behaviours lead to different ice shapes. Despite the complexity of the phenomenon and the dependence on many parameters, existing models are reasonably accurate in predicting both ice shapes and the quantity of ice produced for small droplets (up to about $40 \mu \mathrm{m}$ ). However, for larger droplets $(40-400 \mu \mathrm{m})$ the current models tend to fail, dramatically over-predicting the amount of ice produced and wrongly predicting its location. This is thought to be due, at least in part, to splashing. When larger droplets hit the thin layer of water on the wing they splash, ejecting droplets back into the airstream. These either escape the wing completely (hence the over-prediction of ice mass) or re-impinge further back on the wing (explaining the incorrect prediction of ice location/shape). See Gent et al. (2000) for a review of the factors contributing to aircraft icing and modelling approaches. Ice build-up can have significant influence on the aircraft aerodynamics; better understanding of the influence of splashing on icing is therefore desirable.

Despite this physical importance, there has been relatively little previous direct theoretical input and suitable physical modelling on droplet impact at One contribution of 19 to a Theme 'New developments and applications in rapid fluid flows'. 
a high Reynolds number into water. Early interest appeared in Worthington (1908), whose book includes many images of splashing after both droplet and solid sphere impacts upon a fluid layer. However, several interesting studies have been made on related aspects both analytically, for example, Korobkin \& Pukhnachov (1988), Wilson (1989) and Howison et al. (1991) who consider solutions at small times after impact for solid-water impacts, and Yarin \& Weiss (1955) and Mundo et al. (1995) who consider differing aspects of droplet impact, and numerically such as in Weiss \& Yarin (1999), who use a Lagrangian-type approach. Much work has also been produced by Josserand \& Zaleski (2003, and references therein), who have developed powerful three-dimensional techniques for simulating droplet impacts, although their parameter ranges differ from those important in an icing context. More recent studies have been completed by Purvis \& Smith (2004a, 2005) and Smith et al. (2003).

Section 2 outlines the central impact problem, which is analysed in $\S 3$ at small distortion times for a droplet entering deep water. Sections 4 and 5 consider instead a thin layer. Section 6 then describes the numerical work to examine $O(1)$ times. Final brief comments are provided in $\S 7$.

\section{Governing equations}

We consider a single water droplet impacting upon an otherwise undisturbed layer of water. The droplet has diameter $D$, the layer has depth $H$ and the incoming droplet velocity is $V$. Non-dimensionalized on a typical droplet diameter (40-400 $\mu \mathrm{m}$ in the large droplet icing context) and incoming velocity (150-200 $\mathrm{m} \mathrm{s}^{-1}$ but can vary), the incident droplet thus has diameter $O(1)$ and $V=-1$. Typical Reynolds numbers are large $O\left(10^{5}\right)$ and so it seems reasonable to treat the problem as inviscid. Surface tension is small, $W e \sim O\left(10^{5}\right)$ and the effect of gravity is also negligible $\left(F r \sim O\left(10^{8}\right)\right)$. We examine the flow in two dimensions and also ignore the influence of air (cf. Purvis \& Smith 2005; Smith et al. 2003). The unsteady two-dimensional Euler equations, namely

$$
\begin{gathered}
U_{x}+V_{y}=0, \\
U_{t}+U U_{x}+V U_{y}=-P_{x}, \\
V_{t}+U V_{x}+V V_{y}=-P_{y},
\end{gathered}
$$

then apply in terms of the velocities $U, V$ in coordinates $x, y$ with time $t$ and pressure $P$. The undisturbed layer lies in $y \leq 0$.

\section{Droplet impacting upon a deep layer}

First, we consider the small-time behaviour of a circular droplet entering an undisturbed horizontal layer of infinite depth. 


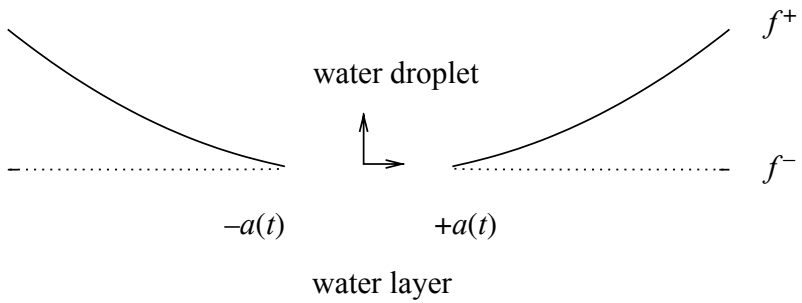

Figure 1. Local problem in neck region, as a droplet descends onto a still water layer.

(a) Small-time behaviour

In a region where $x$ and $y$ are $O\left(t^{1 / 2}\right)$ around the neck of the impact, the velocities, pressure and free-surface height scale as

$$
[U, V, P, F] \sim\left[u, v, t^{-(1 / 2)} p, t f\right]
$$

and substitution into the governing Euler equations yields the Cauchy-Riemann equations for $f_{t t}$ and $-p_{x}$ (extended into the $x-y$ plane),

$$
f_{t t x}^{ \pm}=-p_{x y}^{ \pm}, \quad f_{t t y}^{ \pm}=p_{x x}^{ \pm}
$$

where 'plus or minus' indicates quantities above (in the droplet) and below (in the layer; see figure 1). The boundary conditions

$$
f^{+} \sim x^{2}-t, \quad f^{-} \rightarrow 0 \quad \text { as } \quad|x| \rightarrow \infty, y=0,
$$

match with the circular droplet impacting with velocity -1 above and with the undisturbed horizontal layer below in the outer $x, y \sim O(1)$ region, while

$$
p^{+}=p^{-}=0 \text { for }|x|>a(t), y=0,
$$

as surface tension has no effect to leading order on this scale (see $\S 3 c$ below), and pressure continuity and equal free-surface shapes require

$$
p^{+}=p^{-}, \quad f^{+}=f^{-}, \quad \text { for }|x|<a(t), y=0 .
$$

Finally, the contact points $x= \pm a(t)$ where the upper and lower free surfaces meet are also unknown in this problem with mixed boundary conditions.

Cauchy's integral formula, however, gives the integral relation

$$
p^{+}+p^{-}=\frac{1}{\pi} \int_{-\infty}^{\infty} \frac{f_{t t}^{+}-f_{t t}^{-}(\xi, t)}{\xi-x} \mathrm{~d} \xi .
$$

For $|x|>a(t)$ the pressures are both zero (condition (3.4)) and the integrand is only non-zero for $|\xi|>a(t)$. Inverting the resulting relation (see Muskhelishvili 1946), integrating twice in $t$ and imposing (3.3)-(3.5) leads to the solutions

$$
f^{ \pm}= \pm \frac{1}{2}|x|\left(x^{2}-2 t\right)^{1 / 2}+\frac{x^{2}}{2}-\frac{t}{2}, \quad x^{2}>2 t ; \quad f^{ \pm}=\frac{x^{2}}{2}-\frac{t}{2}, \quad x^{2}<2 t .
$$

The contact points are thus at $x= \pm(2 t)^{1 / 2}$. The small-time solution $(3.7)$ is shown in figure 2 for two times; as time increases, the contact point in the neck 


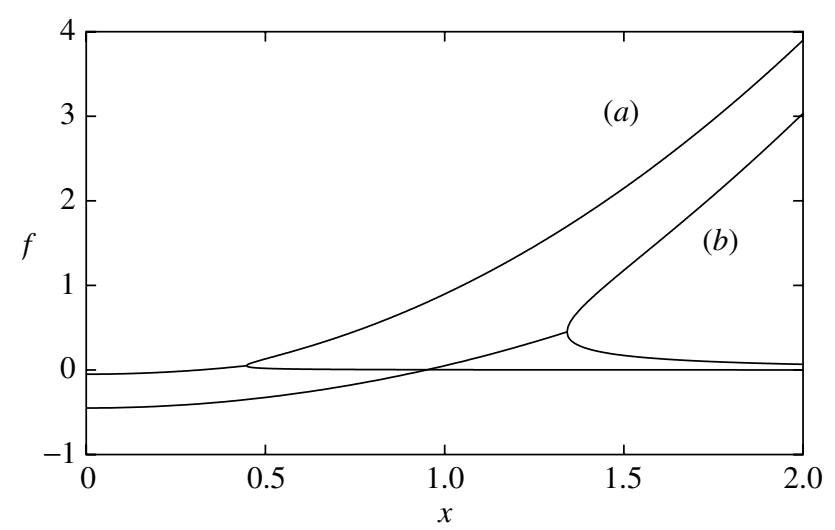

Figure 2. The small-time solution at two times: $(a) t=0.1$, (b) $t=0.9$.

region where the upper and lower free surfaces meet moves outwards and upwards.

The pressure in the connected region $|x|<(2 t)^{1 / 2}$ follows from $[f]_{t t}$ in $(3.7)$ and $p_{x}^{+}=p_{x}^{-}$in (3.5), so that (3.6) becomes

$$
p_{x}^{ \pm}=-\frac{x}{4 \pi} \int_{\sqrt{2 t}}^{\infty} \frac{\xi}{\left(\xi^{2}-2 t\right)^{3 / 2}} \frac{\mathrm{d} \xi}{\xi^{2}-x^{2}},
$$

giving

$$
p^{ \pm}=-\frac{1}{4}\left(2 t-x^{2}\right)^{-(1 / 2)} .
$$

(Similar results hold for a non-flat layer incidentally.) To smooth out the squareroot behaviour exhibited by (3.7) and the singular behaviour of the pressure near the contact point a smaller region arises where

$$
(x-\sqrt{2 t}, y, U, V, P, F)=\left(t^{3 / 2} \bar{x}, t^{3 / 2} \bar{y}, t^{1 / 2} \bar{u}, t^{1 / 2} \bar{v}, t^{-1} \bar{p}, t^{3 / 2} \bar{f}\right)+\ldots
$$

This expansion leads locally to the steady Euler equations. The problem is exactly that encountered in solid-water impacts and the solution is given in Howison et al. (1991). It is found that a fast-moving horizontal jet appears in the neck region. This response is the limiting case of that set out in $\S 5$ as $h \rightarrow \infty$.

\section{(b) Impact at incidence}

The solution outlined above assumes a normal impact. This can be easily extended to allow for oblique impact. The form for the pressure and streamfunction from the direct impact solution has

$$
p^{+}-\mathrm{i} \psi_{t}^{+}=\frac{\mathrm{i}}{4}\left(z^{2}-2 t\right)^{-(1 / 2)}
$$


with $z=x+\mathrm{i} y$. Integrating with respect to $t$ leads to

$$
q^{+}-\mathrm{i} \psi^{+}=-\frac{\mathrm{i}}{4}\left(z^{2}-2 t\right)^{1 / 2}+\mathrm{i} \hat{c} z
$$

where $q_{t}^{+}=p^{+}$and the arbitrary function is determined by insisting $|z| \sim t^{1 / 2}$. The imaginary part of (3.12) at large distances gives $\psi^{+} \sim\left((1 / 2)-c_{\mathrm{r}}\right) x+c_{\mathrm{i}} y$, where $\hat{c}=c_{\mathrm{r}}+\mathrm{i} c_{\mathrm{i}}$. To ensure $v \rightarrow-1$ in the farfield we need $c_{\mathrm{r}}=-1 / 2$. We can, however, choose $c_{\mathrm{i}}$ to give the required horizontal velocity of the droplet. Since $f_{t}=-\psi_{x}$ we find from (3.12) that

$$
f_{t}^{+}=-\psi_{x}^{+}=-\frac{1}{2}|x|\left(x^{2}-2 t\right)^{-(1 / 2)}-\frac{1}{2},
$$

which, on integration in $t$, gives the previous result (3.7). Note that this is independent of the initial horizontal velocity $c_{\mathrm{r}}$. The water layer solution remains unchanged, i.e. $c_{\mathrm{i}}^{-}=0, \quad c_{\mathrm{r}}^{-}=0$, leaving a jump in $u$ across $y=0,|x|<(2 t)^{1 / 2}$, according to this model.

Consideration of the inner jetting region discussed for the normal impact above shows that this sideways motion does not affect the local jetting to leading order. So the small-time solution holds for oblique impacts also. Similar phenomena are found in stone skimming and solid-liquid impact (Howison et al. 2003), where the horizontal velocity there also does not affect the leading-order solution at small times unless the horizontal velocity is sufficiently large, of the order of the initial jetting velocity, and likewise in air-water interactions with a moving wall (Smith et al. 2003).

\section{(c) Surface tension}

To include surface tension in the small-time model, consider a region near contact where $(x-\sqrt{2 t}, y)=t^{m}(\bar{x}, \bar{y})$ and expand

$$
(U, V, P, F)=\left(t^{-(m / 2)+1 / 4} \bar{u}, t^{-(m / 2)+1 / 4} \bar{v}, t^{-(m / 2)-(1 / 4)} \bar{p}, t^{m / 2+3 / 4} \bar{f}\right)+\ldots,
$$

where the $F$ scaling is from $F$ being $O(t)$ in the $x \sim y \sim t^{1 / 2}$ region but parabolic near the contact point. Then the $V$ scaling follows from $V \sim F_{t}, U$ from continuity and the $P$ condition from balancing $V_{t}$ with $P_{y}$. This leaves $F_{x x} \sim t^{-(3 m / 2)+(3 / 4)}$. Comparing this with the pressure $P \sim t^{-(m / 2)-(1 / 4)}$ then, as surface tension is proportional to the curvature of the free surface, the surface-tension effect enters where $F_{x x} \sim P$, i.e. where $m=1$. So surface tension has a substantial influence on a scale intermediate between the $t^{1 / 2}$ region and the initial jetting.

Substituting (3.14) into the Euler equations yields to leading order

$$
\bar{u}_{\bar{x}}+\bar{v}_{\bar{y}}=0, \quad-\frac{\sqrt{2}}{2} \bar{u}_{\bar{x}}=-\bar{p}_{\bar{x}}, \quad-\frac{\sqrt{2}}{2} \bar{v}_{\bar{x}}=-\bar{p}_{\bar{y}} .
$$

Scaling out the $\sqrt{2} / 2$ factor and combining (3.15) leaves the Cauchy-Riemann equations for $\bar{v}, \bar{p}$. These are to be solved subject to

$$
\bar{p}=\sigma \bar{f}_{\bar{x} \bar{x}}, \bar{v}=-\bar{f}_{\bar{x}} \quad \text { for } \quad \bar{x}>0, \quad \bar{v}=0 \quad \text { for } \quad \bar{x}<0,
$$




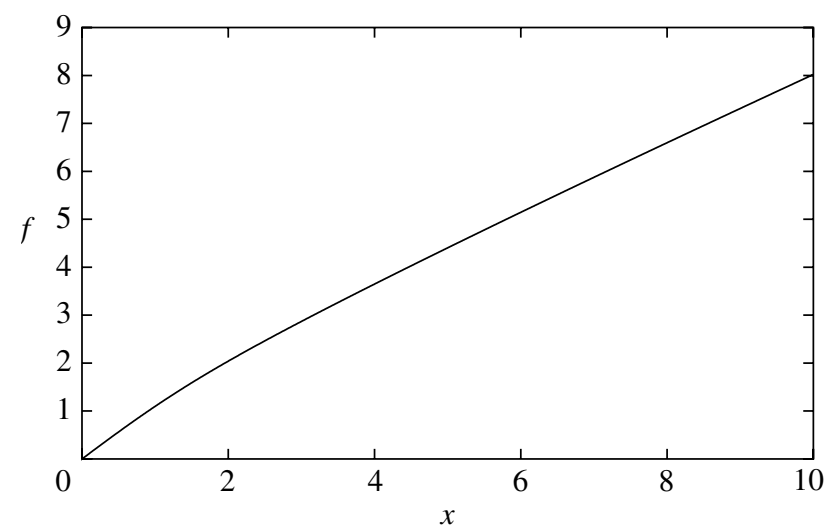

Figure 3. The numerical solution of the surface tension integral equation.

where $\sigma$ is the non-dimensionalized coefficient of surface tension. Cauchy's integral formula now leads to the integral equation

$$
\sigma \bar{f}_{\bar{x} \bar{x}}=\frac{1}{\pi} \int_{0}^{\infty} \frac{\bar{f}_{\xi}}{\xi-\bar{x}} \mathrm{~d} \xi
$$

The solution can be obtained numerically or using a Wiener-Hopf approach, as in Stewarton (1960) for a similar equation, and is presented in figure 3. The inclusion of surface tension smooths out the square-root behaviour in $f$ near the contact point, and a linear behaviour can be seen. The influence that this has on the inner jetting region remains unclear however.

\section{Droplet impacting upon a shallow layer}

For thin layers, and as the size of the connected region grows in time, the ground effect may enter the small-time evolution. This occurs when the $t^{1 / 2}$ extent grows as large as the layer depth $h$, i.e. at times $O\left(h^{2}\right)$. The governing equations are then unchanged, as are the boundary conditions. The difference is that we now have an additional requirement on the wall that $v=0$ at the scaled distance $h$ beneath the free surfaces. Introducing the image reduces the problem to solving (3.2) subject to (3.3)-(3.5) but applied both at $y=0$ and at $y=-2 h$.

\section{(a) Integral formulation}

The problem is that of finding the function $w(x+\mathrm{i} y, t)=p_{x}(x, y, t)+\mathrm{i} f_{t t}(x, y, t)$, analytic in the complex plane and bounded in the far field such that

$$
w(x \pm 0 \mathrm{i})=p_{x}^{ \pm}(x)+\mathrm{i} f_{t \bar{t}}^{ \pm}(x), \quad w(x-2 h \mathrm{i} \mp 0 \mathrm{i})=p_{x}^{ \pm}(x)-\mathrm{i} f_{t t}^{ \pm}(x),
$$

where the values on the free surfaces, $f_{t \bar{t}}^{ \pm}(x, t)$ and $p_{x}^{ \pm}(x, t)$, are either known from (3.3) to (3.5) or unknown. A method similar to that in Jones (2000) and Purvis \& Smith (2004b), based on applying Cauchy's integral formula, yields integral equations for the free surfaces and pressures. The boundary conditions show that 
$p_{x}^{+}-p_{x}^{-}=0$ on the free surface $|x|>a(t)$ since $p^{ \pm}=0$ and also in the middle since $p^{+}=p^{-}$. Likewise, $f_{t t}^{+}-f_{t t}^{-}=0$ for $|x|<a(t)$. Once again, the position of the contact points $x= \pm a(t)$ is unknown in advance. Therefore, the obtained integral equations immediately reduce to

$$
\begin{gathered}
\left\langle f_{t t}\right\rangle=\frac{1}{\pi} \int_{\mathrm{FS}} \frac{2 h}{(\xi-x)^{2}+4 h^{2}}\left[f_{t t}\right](\xi) \mathrm{d} \xi, \\
\left\langle p_{x}\right\rangle=\frac{1}{\pi} \int_{\mathrm{FS}}\left(\frac{1}{\xi-x}+\frac{\xi-x}{(\xi-x)^{2}+4 h^{2}}\right)\left[f_{t t}\right](\xi) \mathrm{d} \xi,
\end{gathered}
$$

where we have introduced the notation $[p]=p^{+}-p^{-},\langle p\rangle=p^{+}+p^{-}$, for the differences and sums of the pressure terms above and below, and similarly for the free-surface shape $f$, and FS denotes the free-surface regions $(-\infty,-a(t)]$ and $[a(t), \infty)$.

We solve these integral equations numerically using a method adapted from Purvis \& Smith (2005). We consider (4.3) for $|x|>a(t)$ when $\left\langle p_{x}\right\rangle=0$ and separate the integral on the right-hand side into a Cauchy part and the remainder. The Cauchy part is then inverted and the resulting equation is evaluated numerically. Results are shown in figure 4 for two values of $h$. Observe that in the large- $h$ case the solution is as before with the contact point moving upward and rightwards. For smaller $h$ the solution initially follows the no-wall case but then the upper free surface is pulled downwards and the free surface of the layer remains largely flat. The numerically determined contact points are very close to $(2 t)^{1 / 2}$ in all the cases that we have examined.

\section{(b) Smaller and larger times}

The behaviour seen in the numerical results can be explained/verified by considering the large and small $h$ limits (or small and large times, respectively). Considering large $h$, the second term in the integrand of equation (4.3) is negligible to leading order and this equation reduces to (3.6), reassuringly the governing equation with no wall present.

The small- $h$ case is not quite so straightforward. A Fourier transform of (4.2), (4.3) leaves

$$
\left\langle\hat{f}_{t t}\right\rangle=\mathrm{e}^{-\beta|w|}\left[\hat{f}_{t t}\right], \quad-\mathrm{i} w\langle\hat{p}\rangle=-\mathrm{isgn}(w)\left(1+\mathrm{e}^{-\beta|w|}\right)\left[\hat{f}_{t t}\right],
$$

where $\beta=2 h$ and 'cap' denotes a transformed quantity. Expanding the sums as $\langle$ cap $\rangle=\langle\text { cap }\rangle_{0}+\beta\langle\text { cap }\rangle_{1}+\ldots$ and the differences similarly, and considering (4.4) for $\beta \ll 1$, we find to leading order

$$
\left\langle\hat{f}_{t t}\right\rangle_{0}=\left[\hat{f}_{t t}\right]_{0}, \quad-\mathrm{i} w\langle\hat{p}\rangle_{0}=-2 \mathrm{isgn}(w)\left[\hat{f}_{t t}\right]_{0} .
$$

The inverse transform then gives the leading-order problem as

$$
\left\langle f_{t t}\right\rangle_{0}=\left[f_{t t}\right]_{0},
$$



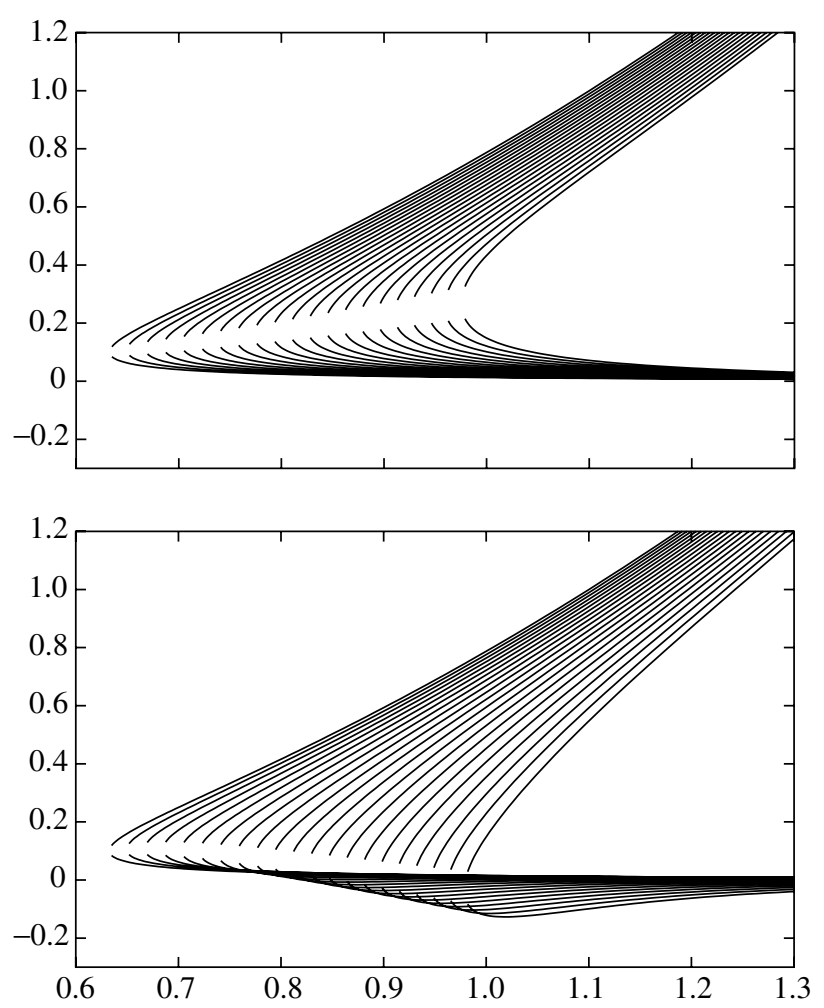

Figure 4. Free surface shapes in the $t \sim h^{2}$ case for $h=10$ (top) and $h=0.2$ (bottom).

$$
\left\langle p_{x}\right\rangle_{0}=\frac{2}{\pi} \int_{-\infty}^{\infty} \frac{\left[f_{t t}\right]_{0}(\xi)}{\xi-x} \mathrm{~d} \xi
$$

Considering (4.6), we find that $f_{t t 0}^{-}=0$ and, as $f^{-} \rightarrow 0$ as $|x| \rightarrow \infty$, then $f_{0}^{-}=0$; the free surface of the layer remains flat to leading order. Now consider (4.7) for $|x|>a(t)$; here $p_{0}^{+}=p_{0}^{-}=0$ and, knowing $f_{0}^{-}$, we find

$$
0=\frac{2}{\pi} \int_{\mathrm{FS}} \frac{f_{t t 0}^{+}(\xi)}{\xi-x} \mathrm{~d} \xi
$$

This is exactly the governing equation for a droplet hitting a solid wall with no water layer present. In summary, to leading order, for $t \gg h^{2}$ the layer remains undisturbed and the droplet reacts as if hitting a wall.

\section{Even larger times}

The next limit of interest is when the wall effect enters the jetting region. This occurs when $h \sim O\left(t^{3 / 2}\right)$, again either for a very thin layer or at later times $O\left(h^{2 / 3}\right)$ as the contact region expands. The governing equations and expansions 


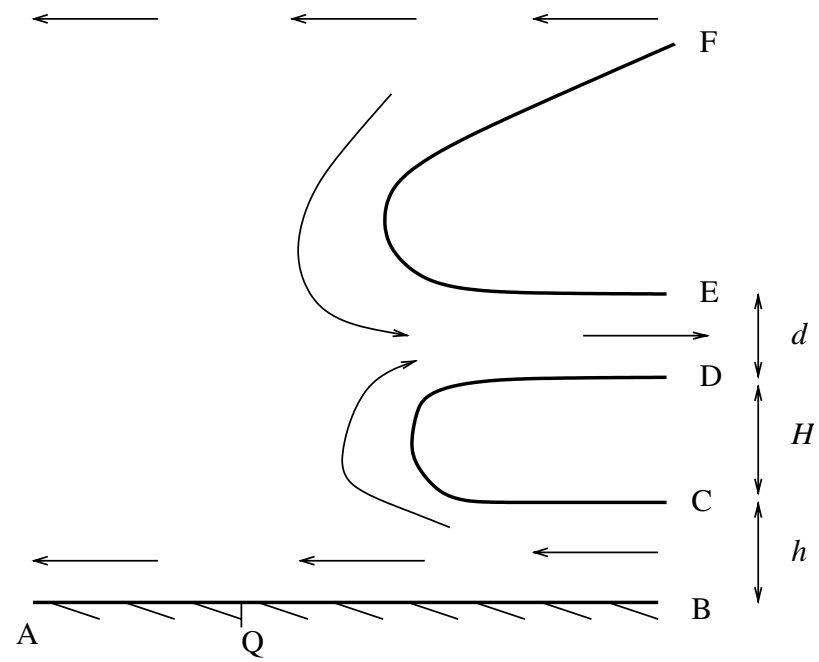

Figure 5. The local $t \sim h^{2 / 3}$ jetting problem. AB is the wall while $\mathrm{CD}, \mathrm{EF}$ are free surfaces.

are unchanged from (3.10), although here we omit the overbars. The problem is set out in figure 5 .

In brief, to find a solution, we consider the domain edge in the $J=u-\mathrm{i} v$ plane which is a circle of radius 1 (the velocity at $\mathrm{F}$ and in the incoming jet $\mathrm{BC}$ is normalized such that $u=-1$ there) but with an extra loop along the real axis from $J=-1$ to the mapped point Q where the minimum velocity is found along the wall, $J=-q$ say. We proceed by mapping this to the upper-half plane $\xi$ via

$$
J=\frac{\left(\xi^{2}-\gamma^{2}\right)^{1 / 2}-\mathrm{i}}{\left(\xi^{2}-\gamma^{2}\right)^{1 / 2}+\mathrm{i}}
$$

where $\gamma=(1-q) /(1+q)$. Now we construct the complex potential $w$. The jet at $\mathrm{BC}$ corresponds to a source in the $\boldsymbol{\xi}$-plane, the jet at DE a sink. Incorporating also the free-stream behaviour at $\mathrm{F}$, ensuring the correct behaviour as $\xi \rightarrow \infty$ and satisfying the requirement that $u$ is a minimum along the wall at $\mathrm{Q}$ leads to the solution

$$
w=\frac{-L^{2}}{4(\xi+\gamma)}+\frac{d-h}{\pi} \ln (\xi+\gamma)+\frac{h}{\pi} \ln (\xi-\gamma),
$$

where $d$ is the width of the jet DE and $h$ is the layer depth. Matching with the (assumed given) upper free-surface shape $f(x)=K x^{1 / 2}$ yields $L^{2}=K^{2} / 8 \gamma$. Additionally, we also need to ensure that the stagnation point in the $\xi$-plane corresponds to the origin in the $J$-plane. Satisfying this requirement implies

$$
w=\frac{-K^{2}}{32 \gamma(\xi+\gamma)}+\frac{K^{2}\left(4 \gamma^{2}-1\right)}{64 \gamma^{2}} \ln (\xi+\gamma)+\frac{K^{2}}{64 \gamma^{2}} \ln (\xi-\gamma) .
$$

Note that the solution yields $h=\pi K^{2} / 64 \gamma^{2}$, determining the value of $\gamma$ for a given layer depth. The total flux into the splash jet $(\xi \rightarrow \infty)$ is also determined 

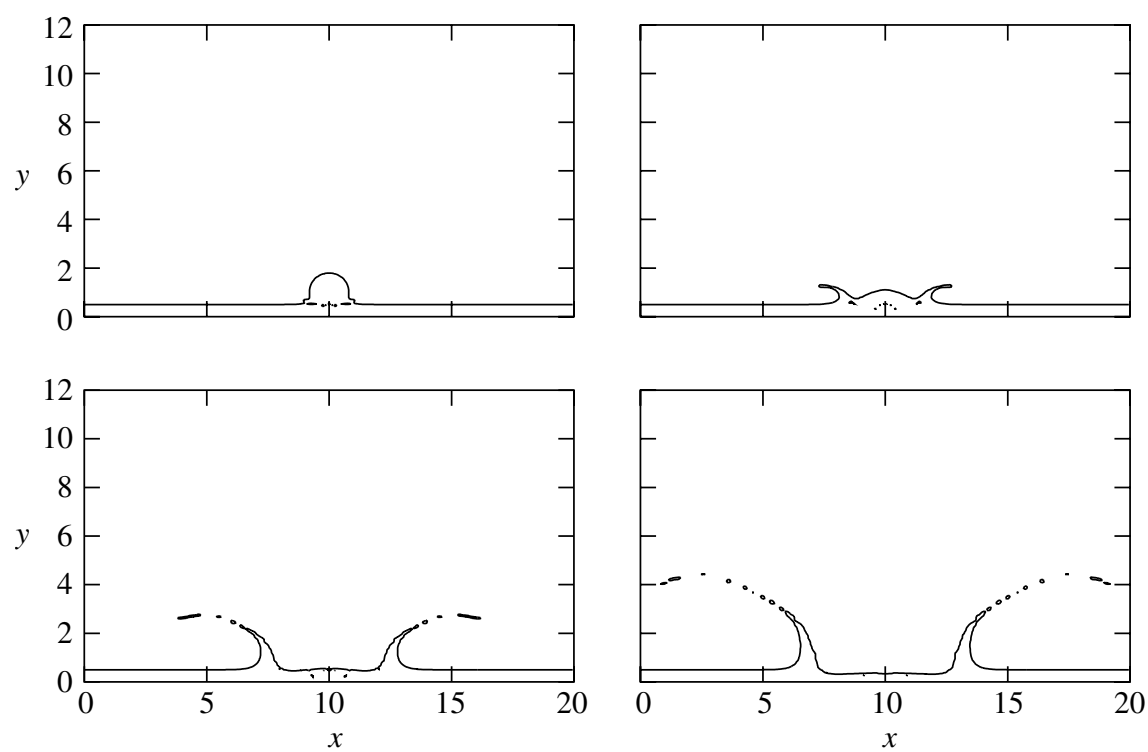

Figure 6. A sample splash generated with VOF method for $D=1.5, H=1 / 2$ at times $t=0.5,1.5,3.5$ and 5.5.

as $d=\pi K^{2} / 16$, which is independent of the layer depth $h$. We assume here that $\gamma<1$ and so there is a single stagnation point in the upper-half plane; similar analysis applies for $\gamma>1$ where there are then two stagnation points on the wall AB.

The detachment $H$, from $\mathrm{C}$ to $\mathrm{D}$, between the inward jet $\mathrm{BC}$ and the emergent jet $\mathrm{DE}$ is related to $\bar{A}$ by an integral in $\xi$ (using $\mathrm{d} z=\mathrm{d} w / J=(\mathrm{d} w / \mathrm{d} \xi)(\mathrm{d} \xi / J)$ ). The imaginary part is

$$
\frac{H}{2}=\int_{\gamma}^{\infty}\left(\frac{L^{2}}{4(E+\gamma)^{2}}+\frac{2 d-h}{2 \pi(E+\gamma)}+\frac{h}{2 \pi(E-\gamma)}\right) \frac{\left(E^{2}-\gamma^{2}\right)^{1 / 2}}{E^{2}-\gamma^{2}+1} \mathrm{~d} E,
$$

which fits with the expectation that $H \rightarrow \infty$ as $\gamma \rightarrow 0$. Smaller times mean $h \rightarrow \infty$ here, when $\gamma \rightarrow 0$, retrieving the Howison et al. (1991) vertically symmetric case. Larger times correspond to $h \rightarrow 0$, when the jet BC reduces, leaving the flow as if onto a flat wall again (compare comments on figure 8 later).

Many details are omitted here, including matching with an outer flow, touchdown of the stagnation point onto the wall and the make-up of the splash jet; a paper on this and other aspects of the small-time behaviour is planned by S. Howison, J. Ockendon, J. Oliver, R. Purvis and F. T. Smith.

\section{Numerical results}

We have also considered the complete problem at $O(1)$ times computationally using a volume-of-fluid (VOF) approach (see Purvis \& Smith (2004a) for the numerical method and more detailed results). We present a few examples to compare with the small-time solution. Figure 6 shows an example of the splash 


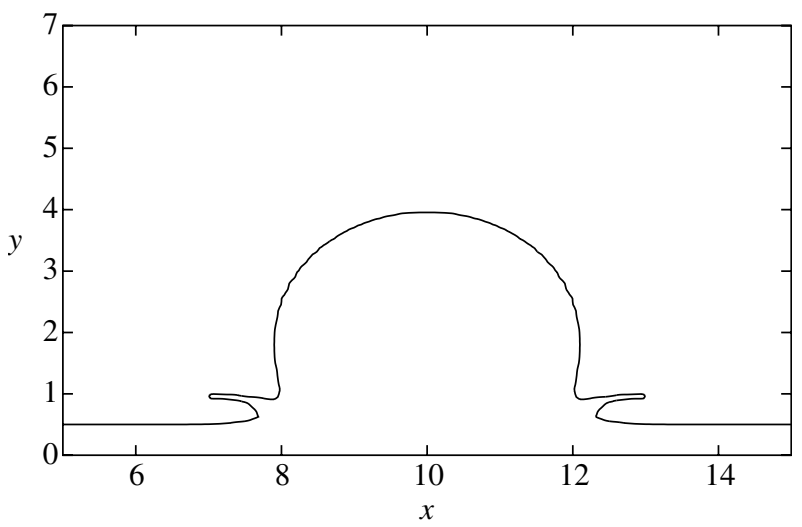

Figure 7. The initial jetting in the VOF solution.
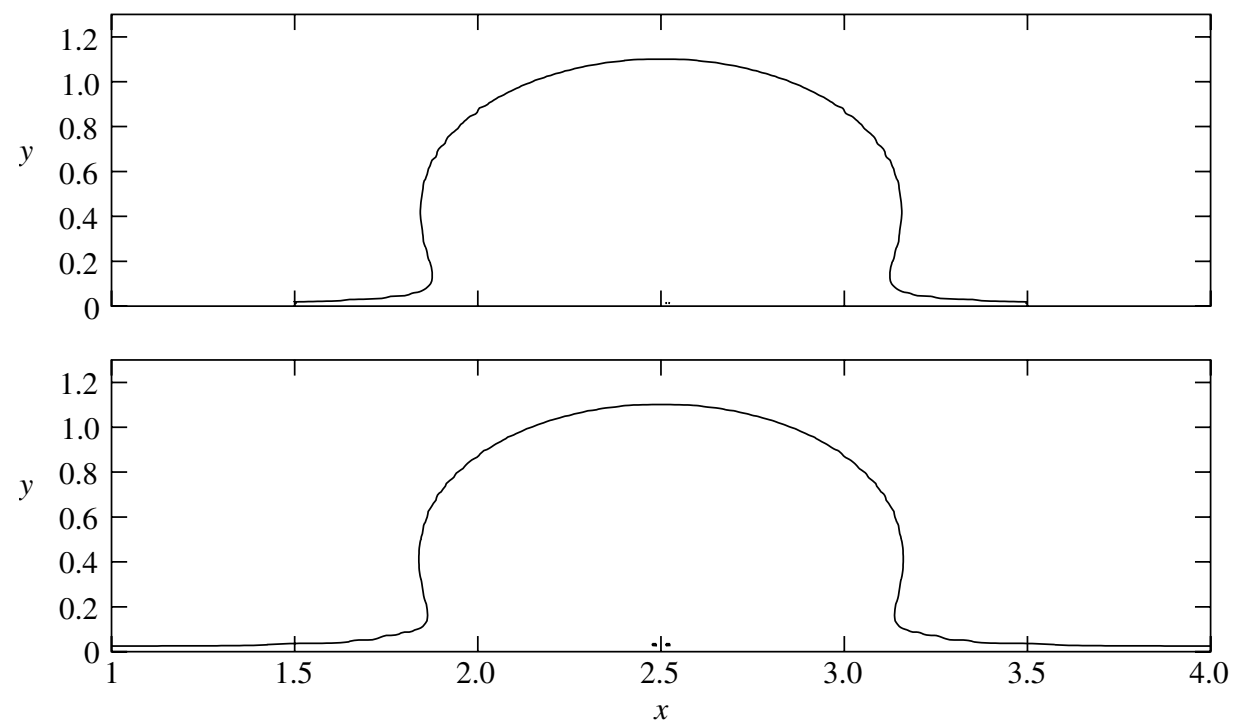

Figure 8. A comparison between the VOF solution of a droplet hitting a wall (top) and a very thin layer (bottom).

produced by a droplet of diameter $D=1.5$ impacting upon a layer of depth $H=0.5$. The droplet starts above the layer and surface tension is included with a physically representative value of the Weber number, $W e=3000$. The numerical method can handle the change in topology as the droplet enters the layer without any special treatment.

Figure 7 shows the free surfaces for a large droplet impact. The jets that can be clearly seen are a continuation of the initial jetting found in $\S 3$. If the numerical method commences from the small-time solution outlined earlier, the results are indistinguishable from starting the droplet above the layer. Favourable comparison with the experimental work of Thoroddson (2002) is also found. 
Finally, figure 8 presents the results for a droplet hitting a dry wall and for a droplet hitting a wall with a very thin layer. As anticipated analytically in $\S \S 4$ and 5 , the layer remains flat and the results for the droplet-wall and dropletthin layer cases are very similar. With deeper layers the jets are forced upwards as in figures 6 and 7 .

\section{Further comments}

This article has examined analytically the small-time behaviour of a droplet impacting upon an undisturbed water layer, for various layer depths, and numerically the $O(1)$-time behaviour. The correspondence between the behaviours predicted thus (as well as the agreement with experiments noted in Purvis \& Smith 2004a) is reassuring, altogether, and suggests continuing both the analysis and the computations. Extra features of interest such as viscosity, density, air flow effects and compressibility need to be incorporated and studied similarly.

We thank EPSRC and QinetiQ for their support through the Faraday Partnership for Industrial Mathematics, managed by the Smith Institute, and also David Allwright, Roger Gent, David Hammond, Richard Moser and Manolo Quero for their interest and helpful discussions. Referees' comments are gratefully acknowledged. We also thank Sasha Korobkin, Sam Howison, John Ockendon and Jim Oliver for their interest and exchanges of information, especially concerning some of the details in $\S 5$.

\section{References}

Gent, R. W., Dart, N. P. \& Cansdale, J. T. 2000 Aircraft icing. Phil. Trans. R. Soc. A 358, 28732911. (doi:10.1098/rsta.2000.0689.)

Howison, S. D., Ockendon, J. R. \& Wilson, S. K. 1991 Incompressible water-entry problems at small deadrise angles. J. Fluid Mech. 222, 215-230.

Howison, S. D., Ockendon, J. R. \& Oliver, J. M. 2003 Oblique slamming, planing and skimming. J. Eng. Math. 48, 321-337.

Jones, M. A. 2000 Mechanisms in Wing-in ground effect aerodynamics. Ph.D. thesis, University of London, UK.

Josserand, C. \& Zaleski, S. 2003 Droplet splashing on a thin liquid film. Phys. Fluids 15, 1650-1657.

Korobkin, A. A. \& Pukhnachov, V. V. 1988 Initial stage of water impact. Ann. Rev. Fluids Mech. 20, 159-185.

Mundo, C., Sommerfeld, M. \& Tropea, C. 1995 Droplet-wall collisions: experimental studies of the deformation and breakup process. Int. J. Multiphase Flow 21, 151.

Muskhelishvili, N. I. 1946 Singular integral equations. Groningen, Holland: Noordhoff.

Purvis, R., Smith, F. T. 2004a. Large droplet impact on water layers. Paper AIAA-2004-414, 42nd Aerospace Sciences Meeting and Exhibit, Reno, NV.

Purvis, R. \& Smith, F. T. $2004 b$ Planar flow past two or more blades in ground effect. Q. J. Mech. Appl. Math. 57, 137-160.

Purvis, R. \& Smith, F. T. 2005 Air-water interactions near droplet impact. Eur. J. Appl. Math. 15, $1-19$.

Smith, F. T., Li, L. \& Wu, G. X. 2003 Air cushioning with a lubrication/inviscid balance. J. Fluid Mech. 482, 291-318.

Stewartson, K. 1960 A note on lifting line theory. Q. J. Mech. Appl. Math. 13, 49-56.

Phil. Trans. R. Soc. A (2005) 
Thoroddson, S. T. 2002 The ejecta sheet generated by the impact of a drop. J. Fluid Mech. 451, 373-381.

Weiss, D. A. \& Yarin, A. 1999 Single drop impact on liquid films: neck distortion, jetting, tiny bubble entrainment and crown formation. J. Fluid Mech. 385, 229-254.

Wilson, S. K. 1989. The mathematics of ship slamming. D.Phil. thesis, Oxford University.

Worthington, A. M. 1908 A study of splashes. London: Longman, Green.

Yarin, A. L. \& Weiss, D. A. 1995 Impact of drops on solid surfaces: self-similar capillary waves, and splashing as a new type of kinematic discontinuity. J. Fluid Mech. 283, 141. 